\title{
Searching for low-mass dark matter via the Migdal effect in COSINE-100
}

G. Adhikari, ${ }^{1}$ N. Carlin, ${ }^{2}$ J. J. Choi, ${ }^{3}$ S. Choi ${ }^{3}$ A. C. Ezeribe, ${ }^{4}$ L. E. França, ${ }^{2}$ C. Ha, ${ }^{5}$ I. S. Hahn, ${ }^{67,8}$ S. J. Hollick, ${ }^{9}$ E. J. Jeon, ${ }^{10}$ J. H. Jo, ${ }^{9}$ H. W. Joo, ${ }^{3}$ W. G. Kang, ${ }^{10}$ M. Kauer ${ }^{11}$ H. Kim, ${ }^{10}$ H. J. Kim, ${ }^{12}$ J. Kim ${ }^{5}$ K. W. Kim ${ }^{10}$ S. H. Kim,${ }^{10}$ S. K. Kim, ${ }^{3}$ W. K. Kim, ${ }^{8,10}$ Y. D. Kim, ${ }^{10,13,8}$ Y. H. Kim, ${ }^{10,14,8}$ Y. J. Ko $\odot,{ }^{10, *}$ H. J. Kwon, ${ }^{8,10}$ D. H. Lee, ${ }^{12}$ E. K. Lee, ${ }^{10}$ H. Lee ${ }^{8,10}$ H. S. Lee ${ }^{10,8, \dagger}$ H. Y. Lee, ${ }^{10}$ I. S. Lee, ${ }^{10}$ J. Lee, ${ }^{10}$ J. Y. Lee, ${ }^{12}$ M. H. Lee, ${ }^{10,8}$ S. H. Lee ${ }^{8,10}$ S. M. Lee, ${ }^{3}$ D. S. Leonard, ${ }^{10}$ B. B. Manzato, ${ }^{2}$ R. H. Maruyama, ${ }^{9}$ R. J. Neal, ${ }^{4}$ S. L. Olsen, ${ }^{10}$ B. J. Park,${ }^{8,10}$ H. K. Park, ${ }^{15}$ H. S. Park, ${ }^{14}$ K. S. Park, ${ }^{10}$ S. D. Park, ${ }^{12}$ R. L. C. Pitta, ${ }^{2}$ H. Prihtiadi, ${ }^{10}$ S. J. Ra, ${ }^{10}$ C. Rott,,${ }^{16,17}$ K. A. Shin, ${ }^{10}$ A. Scarff, N. J. C. Spooner, ${ }^{4}$ W. G. Thompson, ${ }^{9}$ L. Yang, ${ }^{1}$ and G. H. Yu ${ }^{16}$

(COSINE-100 Collaboration)

\author{
${ }^{1}$ Department of Physics, University of California San Diego, La Jolla, California 92093, USA \\ ${ }^{2}$ Physics Institute, University of São Paulo, 05508-090, São Paulo, Brazil \\ ${ }^{3}$ Department of Physics and Astronomy, Seoul National University, Seoul 08826, Republic of Korea \\ ${ }^{4}$ Department of Physics and Astronomy, University of Sheffield, Sheffield S3 7RH, United Kingdom \\ ${ }^{5}$ Department of Physics, Chung-Ang University, Seoul 06973, Republic of Korea \\ ${ }^{6}$ Department of Science Education, Ewha Womans University, Seoul 03760, Republic of Korea \\ ${ }^{7}$ Center for Exotic Nuclear Studies, Institute for Basic Science (IBS), Daejeon 34126, Republic of Korea \\ ${ }^{8}$ IBS School, University of Science and Technology (UST), Daejeon 34113, Republic of Korea \\ ${ }^{9}$ Department of Physics and Wright Laboratory, Yale University, New Haven, Connecticut 06520, USA \\ ${ }^{10}$ Center for Underground Physics, Institute for Basic Science (IBS), Daejeon 34126, Republic of Korea \\ ${ }^{11}$ Department of Physics and Wisconsin IceCube Particle Astrophysics Center, \\ University of Wisconsin-Madison, Madison, Wisconsin 53706, USA \\ ${ }^{12}$ Department of Physics, Kyungpook National University, Daegu 41566, Republic of Korea \\ ${ }^{13}$ Department of Physics, Sejong University, Seoul 05006, Republic of Korea \\ ${ }^{14}$ Korea Research Institute of Standards and Science, Daejeon 34113, Republic of Korea \\ ${ }^{15}$ Department of Accelerator Science, Korea University, Sejong 30019, Republic of Korea \\ ${ }^{16}$ Department of Physics, Sungkyunkwan University, Suwon 16419, Republic of Korea \\ ${ }^{17}$ Department of Physics and Astronomy, University of Utah, \\ Salt Lake City, Utah 84112, USA
}

(Received 12 October 2021; accepted 7 January 2022; published 22 February 2022)

\begin{abstract}
We report on the search for weakly interacting massive particle (WIMP) dark matter candidates in the galactic halo that interact with sodium and iodine nuclei in the COSINE-100 experiment and produce energetic electrons that accompany recoil nuclei via the Migdal effect. The WIMP mass sensitivity of previous COSINE-100 searches that relied on the detection of ionization signals produced by target nuclei recoiling from elastic WIMP-nucleus scattering was restricted to WIMP masses above $\sim 5 \mathrm{GeV} / c^{2}$ by the detectors' 1 keVee energy-electron-equivalent threshold. The search reported here looks for recoil signals enhanced by the Migdal electrons that are ejected during the scattering process. This is particularly effective for the detection of low-mass WIMP scattering from the crystals' sodium nuclei in which a relatively larger fraction of the WIMP's energy is transferred to the nucleus recoil energy and the excitation of its orbital electrons. In this analysis, the low-mass WIMP search window of the COSINE-100 experiment is extended to WIMP mass down to $200 \mathrm{MeV} / \mathrm{c}^{2}$. The low-mass WIMP sensitivity will be further improved by lowering the analysis threshold based on a multivariable analysis technique. We consider the influence of these improvements and recent developments in detector performance to reevaluate sensitivities for the future COSINE-200 experiment. With a 0.2 keVee analysis threshold and
\end{abstract}

yjko@ibs.re.kr

thyunsulee@ibs.re.kr

Published by the American Physical Society under the terms of the Creative Commons Attribution 4.0 International license. Further distribution of this work must maintain attribution to the author(s) and the published article's title, journal citation, and DOI. Funded by $S C O A P^{3}$. 
high light-yield $\mathrm{NaI}(\mathrm{Tl})$ detectors (22 photoelectrons/keVee), the COSINE-200 experiment can explore low-mass WIMPs down to $20 \mathrm{MeV} / c^{2}$ and probe previously unexplored regions of parameter space.

DOI: 10.1103/PhysRevD.105.042006

\section{INTRODUCTION}

A number of astrophysical observations provide evidence that the dominant matter component of the Universe is not ordinary matter, but rather nonbaryonic dark matter $[1,2]$. Theoretically favored dark matter candidates are weakly interacting massive particles (WIMPs) [3,4]. Many direct searches for WIMP dark matter in deep underground laboratories have been performed and have yet to find a signal [5-11]. In light of the absence of signal in the WIMP dark matter mass range of $\mathrm{GeV} / c^{2}$ to $\mathrm{TeV} / c^{2}$, there is an increasing interest in low-mass dark matter particles in the sub-GeV $/ c^{2}$ mass range [12-19].

In this paper, we report on low-mass dark matter searches for WIMP-nuclei interactions by looking for electron recoils induced from secondary radiation via the Migdal process [20,21] in COSINE-100 data. Data used for COSINE-100 searches has a 1 keVee analysis threshold $[22,23]$, where the unit keVee is the electron recoilequivalent energy in kiloelectron volts. Since the total energy in the Migdal electron and the nuclear recoil is larger than the deposited energy of typical elastic nuclear recoil, our searches are extended to WIMP masses as low as $200 \mathrm{MeV} / c^{2}$.

In the future, this search can be enhanced by lowering the analysis threshold through multivariable analysis or deep machine learning techniques, as discussed in Sec. V, where we evaluate sensitivities of the COSINE-200 experiment, which will have lower analysis threshold, reduced internal background by controlled crystal growth [24] and improved light yield using a novel encapsulation method [25].

\section{EXPERIMENT}

The COSINE-100 experiment [26] is installed in the Yangyang underground laboratory (Y2L) utilizing the space provided by the Yangyang pumped storage power plant in South Korea [27,28]. The laboratory is located at a vertical depth of $700 \mathrm{~m}$ that provides a water-equivalent overburden of $1800 \mathrm{~m}$ [29]. A 2-km-long driveway provides access to the laboratory as well as air ventilation. The laboratory is equipped with a cleanroom and an air-conditioning system providing a low dust level, and constant temperature and humidity of $24.21 \pm 0.06^{\circ} \mathrm{C}$ and $36.7 \pm 1.0 \%$, respectively $[26,30]$. The contamination level of ${ }^{222} \mathrm{Rn}$ in the room is measured to $36.7 \pm 5.5 \mathrm{~Bq} / \mathrm{m}^{3}$. The readout electronics, high voltages that are applied to photomultiplier tubes (PMTs), and data acquisition system are also monitored and stably maintained [30].
The COSINE-100 detector, shown in Fig. 1, consists of a $106 \mathrm{~kg}$ array of eight ultrapure $\mathrm{NaI}(\mathrm{Tl})$ crystals each coupled to two PMTs. The crystal array is immersed in an active veto detector comprised of 2,200 L of linear alkylbenzene (LAB)-based liquid scintillator (LS) to attenuate or tag the influence of external or internal radiations [31,32]. The LAB-LS is contained within a box made with $1 \mathrm{~cm}$ thick acrylic and $3 \mathrm{~cm}$ thick oxygenfree copper that is surrounded by a $20 \mathrm{~cm}$ thick lead shield. An outer array of plastic scintillation counters is used to tag and veto cosmic-ray muons $[29,33]$.

An event is triggered when coincident single photoelectrons in both PMTs that are coupled to a single crystal are observed within a $200 \mathrm{~ns}$ time window. If at least one crystal satisfies the trigger condition, data from all crystals and the LAB-LS are recorded. The signals from the crystal PMTs are processed by $500 \mathrm{MHz}$ flash analog-to-digital converters and are $8 \mu \mathrm{s}$ long waveforms that start $2.4 \mu \mathrm{s}$ before the trigger. The LAB-LS and plastic scintillator signals are processed by charge-sensitive flash analog-todigital converters. Muon events are triggered by coincident signals from at least two plastic scintillators. The LAB-LS signals do not generate triggers, except in the case of energetic muon events that are coincident with one of the muon detector panels. A trigger and clock board read the trigger information from individual boards and generate a global trigger and time synchronizations for all of the

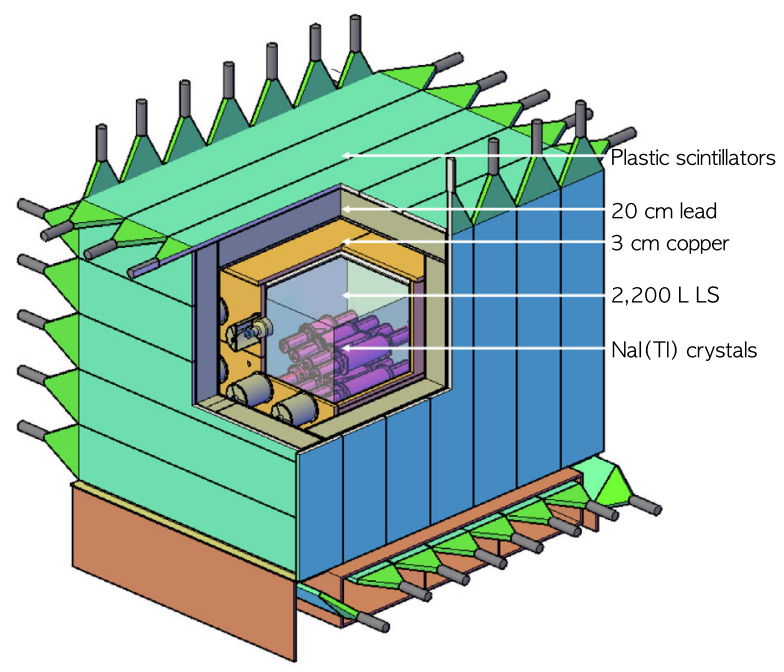

FIG. 1. Schematic of the COSINE-100 detector. The NaI(Tl) $(106 \mathrm{~kg}$ ) detectors are immersed in the 2,200 L LAB-LS that is surrounded by layers of copper and lead shielding. 
modules. Details of the COSINE-100 data acquisition system are described in Ref. [34].

\section{MIGDAL EFFECT}

Direct detection of WIMP dark matter with mass below sub-GeV/ $/ c^{2}$ is limited by an energy threshold of the detector in the range about 0.1-1 keVee. Because the nuclear recoil energies from WIMP-nuclei interactions are quenched (the scintillation signals from nuclear recoils are only an order of $10 \%$ of the signals from the same energy deposition of electrons [35-38]), this energy range corresponds to $1-10 \mathrm{keVnr}$, where $\mathrm{keVnr}$ is kiloelectron volt nuclear recoil energy. The WIMP-nucleus interaction used in typical direct detection searches assumes that the electron cloud is tightly bound to the nucleus and that the orbit electrons remain in stable states. However, energy transferred to nuclei after collision may lead to excitation or ionization of atomic electrons via the Migdal process [20,21]. This process can lead to the production of energetic electron-induced signals that are produced in association with the primary nuclear recoil. For a WIMPnucleus interaction, even if the electron equivalent energy implied by the quenching factor is below the energy threshold of the detector, these Midgal-effect secondary electrons can produce electron equivalent energy that is above the threshold, making detectors sensitive to sub$\mathrm{GeV} / c^{2}$ dark matter interactions. Several experimental groups have already exploited this effect to search for dark matter with sub- $\mathrm{GeV} / c^{2}$ masses [39-43].

The differential nuclear recoil rate per unit target mass for elastic scattering between WIMPs of mass $m_{\chi}$ and target nuclei of mass $M$ is [44]

$$
\frac{d R_{\mathrm{nr}}}{d E_{\mathrm{nr}}}=\frac{\rho_{\chi}}{2 m_{\chi} \mu^{2}} \sigma\left(M, E_{\mathrm{nr}}\right) \int_{v>v_{\min }} d^{3} v f(\mathbf{v}, t),
$$

where $\rho_{\chi}$ is the local dark matter density, $E_{\mathrm{nr}}$ is the nuclear recoil energy, $\sigma\left(M, E_{\mathrm{nr}}\right)$ is the WIMP-nucleus cross section and $f(\mathbf{v}, t)$ is the time-dependent WIMP velocity distribution. The reduced mass $\mu$ is defined as $m_{\chi} M /\left(m_{\chi}+M\right)$ and the minimum WIMP velocity $v_{\min }$ is $\sqrt{M E_{\mathrm{nr}} / 2 \mu^{2}}$.

The rate of ionization due to the Migdal effect for a nuclear recoil energy $E_{\mathrm{nr}}$ accompanied by an ionization electron with energy $E_{\mathrm{ee}}$ is given by the nuclear recoil in Eq. (1) multiplied by the ionization rate [21],

$$
\begin{aligned}
\frac{d R}{d E_{\mathrm{ee}}}= & \int d E_{\mathrm{nr}} d v \frac{d^{2} R}{d E_{\mathrm{nr}} d v} \\
& \times \frac{1}{2 \pi} \sum_{n, l} \frac{d}{d E_{\mathrm{nr}}} p_{q_{e}}^{c}\left(n, l \rightarrow E_{\mathrm{ee}}-E_{n, l}\right) .
\end{aligned}
$$

Here $p_{q_{e}}^{c}$ is the probability for an atomic electron with quantum number $(n, l)$ and binding energy $E_{n, l}$ to be

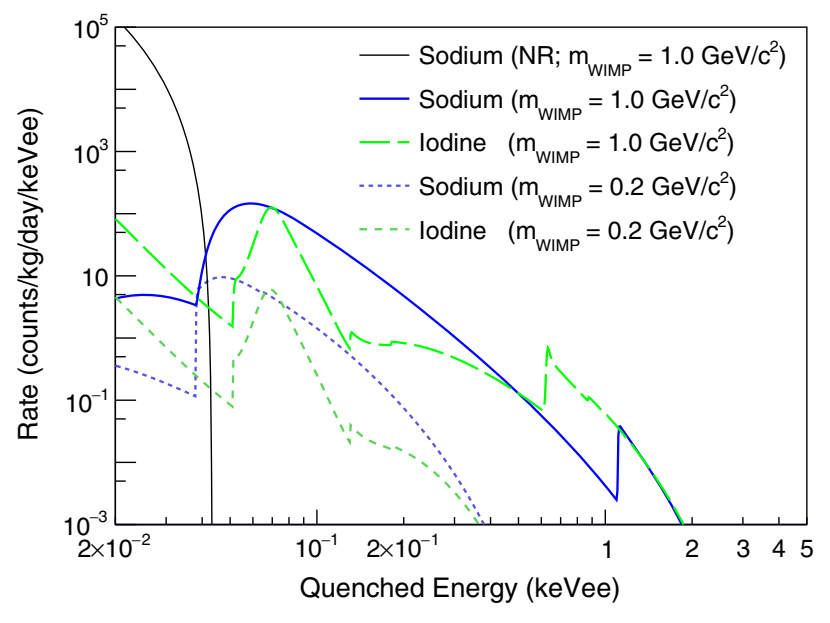

FIG. 2. Expected signals without energy resolution from WIMP-nuclei spin-independent (SI) interaction via the Migdal process are presented for WIMP masses of 0.2 and $1.0 \mathrm{GeV} / c^{2}$ assuming WIMP-nucleon SI cross section of $1 \mathrm{pb}$. Sodium and iodine spectra produced by the Migdal effect are separately presented and compared with the nuclear recoil spectrum of WIMP-sodium interaction for $1.0 \mathrm{GeV} / c^{2}$ WIMP mass. Here the nuclear recoil energy is quenched to the electron recoil energy using the measured quenching factor reported in Ref. [37]. Although the nuclear recoil deposit is below the 1 keVee energy threshold, the electron energy from the Migdal effect can produce above-threshold signals.

ejected with a kinetic energy $E_{\mathrm{ee}}-E_{n, l}$, and $q_{e}$ is the electron momentum in the nucleus rest frame. When the shell vacancy is refilled, an $\mathrm{x}$ ray or an auger electron with energy $E_{n, l}$ is emitted. This takes into account the fact that the emitted electron may come from an inner orbital and the remaining excited state will release additional energy when it returns to its ground state. The differential probability rates for sodium and iodine were calculated in Ref. [21]. Figure 2 shows the differential ionization rates as a function of the electron recoil energy $E_{\text {ee }}$ for sodium and iodine nuclei considering two different WIMP masses.

This description assumes isolated atomic targets that interact with WIMP particles [21,45], which for inner-shell electrons provided a correct estimate of the expected signal rate. For outer electrons, the complicated electronic band structure and crystal form factor can affect the rate for the Migdal effect and significantly improved sensitivity in semiconductors has been reported in Refs. [46,47]. In this analysis, we follow the atomic target approximation because at our analysis threshold, inner shell electrons are the dominant contributors to the Migdal process.

\section{DATA ANALYSIS}

We use data obtained from October 2016 to July 2018, corresponding to 1.7 years exposure that were used for our first annual modulation search [48] and the modeldependent WIMP dark matter search using the energy 
spectra [23]. During the 1.7 years data-taking period, no significant environmental anomalies or unstable detector performance were observed. Six of the eight crystals have a high light yield of approximately $15 \mathrm{NPE} / \mathrm{keVee}$, where NPE corresponds to the number of photoelectron, and enables an analysis threshold of 1 keVee. The other two crystals had lower light yields and required higher analysis thresholds $[26,49]$. Since their direct impact on the lowenergy signal search is not substantial, we do not include these two crystals in this analysis.

In the off-line analysis, muon-induced events are rejected when the crystal hit events and muon candidate events in the muon detector $[29,33]$ are coincident within $30 \mathrm{~ms}$. Additionally, we require that the leading edges of the trigger pulses start later than $2.0 \mu \mathrm{s}$ after the start of the recording, that the waveforms from the hit crystal contain more than two single photoelectrons and the integral waveform area below the baseline does not exceed a limit. These criteria reject muon-induced phosphor events and electronic interference events. A multiple-hit event is one in which more than one crystal has a signal with more than four photoelectrons in an $8 \mu$ s time window or has an LS signal above an $80 \mathrm{keVee}$ threshold within $4 \mu$ s of the hit crystal [32]. A single-hit event is classified as one where only one crystal is hit and none of the other detectors meet the above criteria.

In the low-energy signal region below 10 keVee, PMT-induced noise events contribute to the single-hit WIMP-search data. Although PMT noise involves complex phenomena that are far from being completely understood, we categorize two distinct classes of the PMT-induced noise events. The first class has a fast decay time of less than $50 \mathrm{~ns}$, compared with typical $\mathrm{NaI}(\mathrm{Tl})$ scintillation of about $250 \mathrm{~ns}$. This class of noise events may be induced by radioactive decays of $\mathrm{U}, \mathrm{Th}$, and $\mathrm{K}$ inside the PMT materials. These decays may generate ultraviolet and/or visible photons inside the PMTs. The second class, which occurs less often than the first, is characterized by slow rise times (about $100 \mathrm{~ns}$ ) and decay times (about $150 \mathrm{~ns}$ ), as described in Refs. [49,50]. This class of noise may be caused by accumulated charge somewhere in the PMT arising from the high voltage and subsequent discharging that produces a flash inside the PMTs. This class of noise events is intermittently produced by certain PMTs. We have developed monitoring tools for data quality verification, including monitoring event rates of the second class of noise. If a crystal has an increased rate due to the second class of noise, the relevant period of data is removed. One crystal detector has this class of noise for the whole datataking period; for the other five detectors the second-class noise-induced events are absent during more than $95 \%$ of the data taking period. The effective data exposure for the five crystals is $97.7 \mathrm{~kg}$ · year.

The first class of PMT noise-induced events is efficiently rejected by a boosted decision tree (BDT)-based multivariable analysis technique [51]. The parameters used in the BDT include the balance of the deposited charge from two PMTs, the ratio of the leading-edge (0-50 ns) to trailingedge (100-600 ns) charge, a mean-time parameter, which is a logarithm of the amplitude weighted average time of the
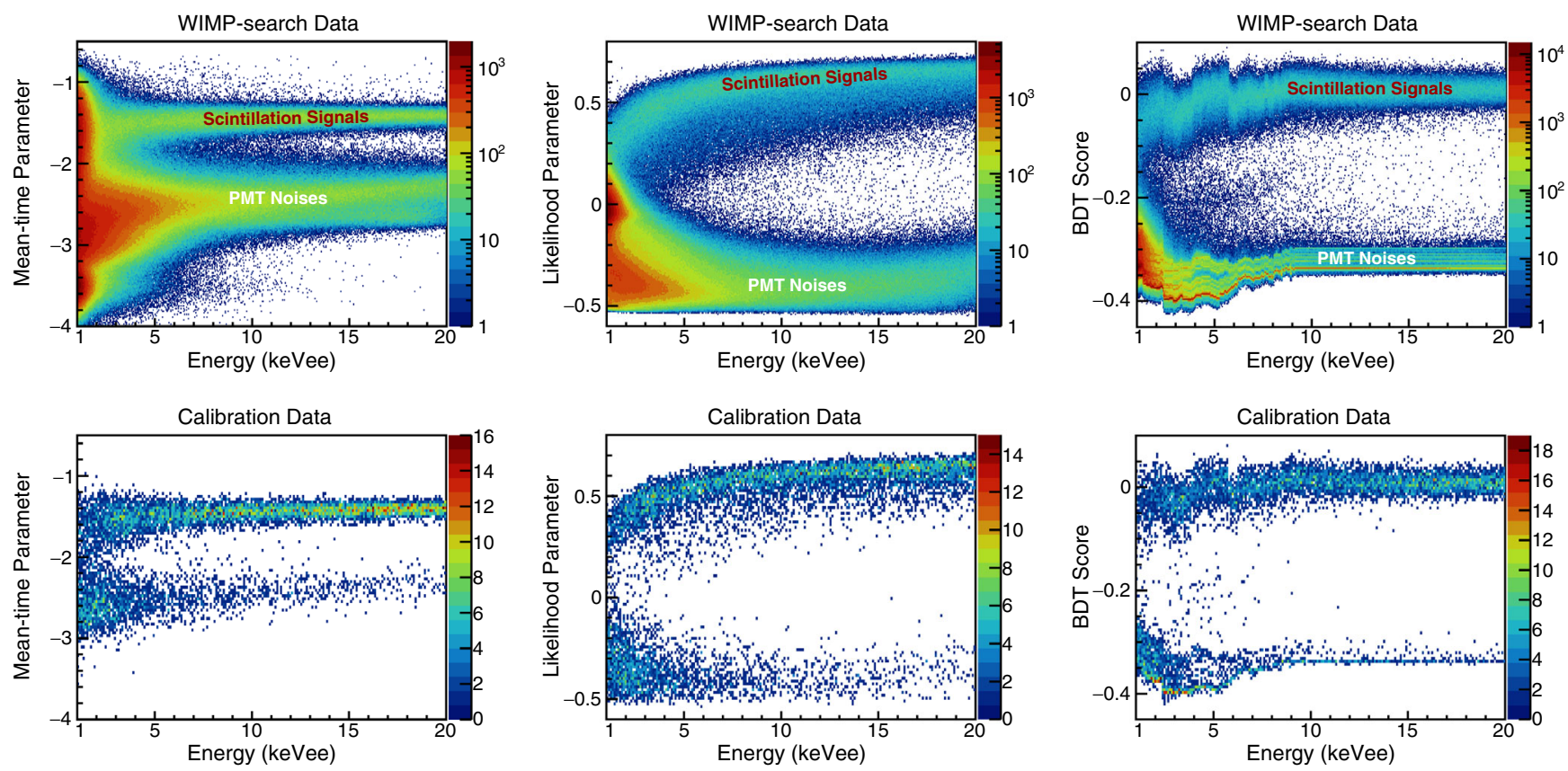

FIG. 3. The distribution of two input parameters, mean-time parameter (left) and the likelihood parameter (center), and output BDT scores (right) are presented separately for the WIMP-search data (top) and ${ }^{60}$ Co calibration data (bottom). Details of all parameters are described in [22]. 
events, and a likelihood parameter for samples of scintillation-signal events and the fast PMT-induced events [22,23]. Figure 3 shows input parameters of mean time and likelihood, as well as output BDT scores for calibration samples and WIMP search data. This procedure reduces the noise contamination level to less than $0.5 \%$ and maintains an $80 \%$ selection efficiency at the lowest energy bin (1-1.25 keVee) can be achieved [22].

GEANT4 [52]-based simulations are used to understand the contribution of each background component [53-55], as well as to verify the energy scales and resolutions. We classify four categories of the NaI-deposited events based on their energies and detector multiplicities. Single-hit and multiple-hit events are further divided into low-energy events 1-70 keVee and high-energy events 70-3000 keVee. The fraction of each background component is determined from a simultaneous fit to the four categorized distributions. For the single-hit events, only 6-3000 keVee events are used to avoid a bias of the signal in the region of interest (ROI). A detailed description of our modeling of the background with the same data is described in Ref. [55].

We consider various sources of systematic uncertainties in the background and signal models. Errors associated with the selection efficiency, the energy resolution, the energy scale, and the background modeling technique are accounted for the shapes of the signal and background probability density functions, as well as in rate changes as described in Ref. [23]. These quantities are allowed to vary within their uncertainties as nuisance parameters in the data fit used to extract the signal.

To estimate the dark matter signal enhancement that is provided by the Migdal effect, we generate signals based on Eq. (2) for various interaction models and different masses in the specific context of the standard WIMP galactic halo model [56,57]. Both a spin-independent (SI) interaction between WIMP and nucleons and a spin-dependent (SD) interaction between WIMP and proton are considered in this analysis. Because both sodium and iodine have nonzero proton spin due to their odd numbers of protons [58,59], $\mathrm{NaI}(\mathrm{Tl})$ detectors are sensitive to the WIMP-proton SD interactions. Responses that include form factors and proton spin values of the nuclei are implemented from the publicly available DMDD package [59-63]. The energy spectra of electron-equivalent energy for the detector are simulated with the energy resolutions of the detectors and the nuclear recoil quenching factors (QFs), where $\mathrm{QF}$ is the ratio of the scintillation light yield from sodium or iodine recoil relative to that from electron recoil for the same energy. We used the QF values from recent measurements with monoenergetic neutron beam [37]. The measurements were modeled using a modified Lindhard formula [64] and described in Ref. [65]. Examples of quenched signal spectra from the Migdal effect of the WIMP-nucleon SI interactions for the WIMP masses of 0.2 and $1.0 \mathrm{GeV} / c^{2}$

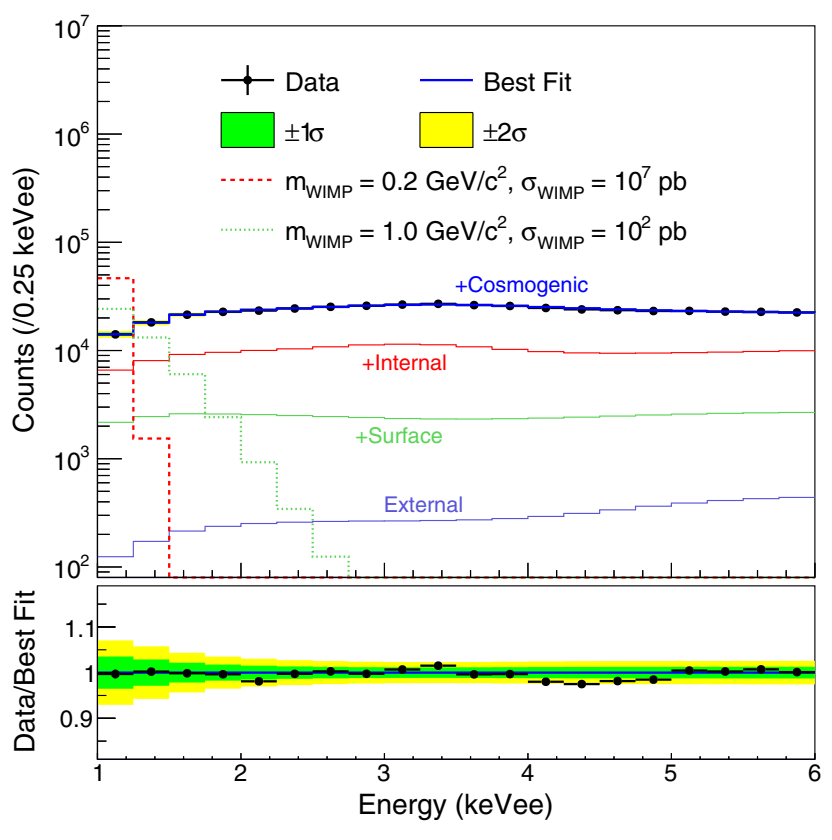

FIG. 4. An example of the fit for a WIMP mass $1.1 \mathrm{GeV} / c^{2}$ using the Migdal effect is presented. The summed energy spectrum for the five crystals (black filled circles) and the best fit (blue solid line) for which no WIMP signals are obtained, are shown together with signal spectra from WIMP masses and SI cross sections of $0.2 \mathrm{GeV} / c^{2}$ and $10^{7} \mathrm{pb}$ (red dashed line), $1.0 \mathrm{GeV} / c^{2}$ and $10^{2} \mathrm{pb}$ (green dotted line). The fitted distribution is broken down into cumulative contributions to the background from external sources, the surface of the crystals and nearby materials, internal radionuclide contaminations, and cosmogenic activation, as indicated. The green (yellow) bands are the $68 \%$ (95\%) C.L. intervals of the systematic uncertainty obtained from the likelihood fit.

are presented in Fig. 2. The output events are subjected to the same selection criteria that are applied to the data.

A Bayesian approach is adopted to extract the WIMP interaction signals using the Migdal effect from the COSINE-100 data. A likelihood function based on Poisson probability is built including constraints that reflect the known levels of the background components. The likelihood fit is applied to the measured single-hit energy spectra between 1 and $6 \mathrm{keVee}$ for each WIMP model with various masses. Each crystal is fitted with a crystal-specific background model and a crystal-correlated dark matter signal. The combined fit is constructed by multiplying likelihoods of the five crystals. The systematic uncertainties are included as nuisance parameters with Gaussian constraints. The same machinery was used for the WIMP dark matter searches using the same dataset but with only the energy from the nuclear recoils without the Migdal effect [23]. An example of the fit for the SI interaction for a WIMP mass of $1.1 \mathrm{GeV} / c^{2}$ is shown in Fig. 4. The summed event spectrum for the five crystals is shown together with the best-fit result. For comparison, the expected signals for WIMP masses (cross 

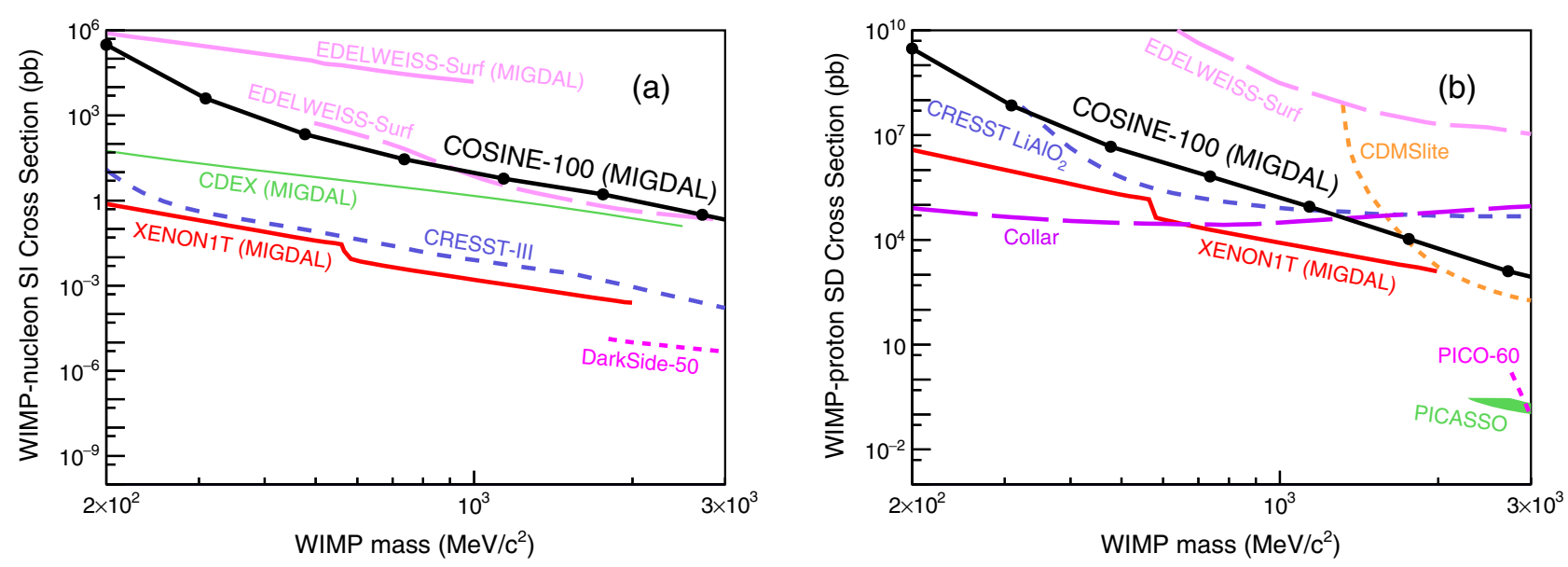

FIG. 5. Limits on WIMP-nucleon SI interaction (a) and WIMP-proton SD interaction (b) induced by the Migdal effect from the COSINE-100 data (black dots) at 90\% C.L. are compared with XENON1T [42], EDELWEISS surface measurement [40], and CDEX [41] with the Migdal effect, and CRESST-III [10], CRESST $\mathrm{LiAlO}_{2} \mathrm{G}$ [66], DarkSide-50 [67], PICASSO [68], CDMSlite [69], EDELWEISS surface [40], Collar [70], and PICO-60 [71].

sections) of $0.2\left(10^{7}\right)$ and $1.0 \mathrm{GeV} / \mathrm{c}^{2}\left(10^{2} \mathrm{pb}\right)$ are presented together. No significant signal for event excess that could be attributed to WIMP interactions is found and $90 \%$ confidence level (C.L.) limits are determined from the marginalization of the likelihood function. Figure 5 shows the $90 \%$ C.L. upper limits from the COSINE-100 data for the WIMP-nucleon SI interactions (a) and the WIMP-proton SD interactions (b) compared with the limits from XENON1T [42], EDELWEISS surface measurement [40], and CDEX [41] using the Migdal effect, as well as CRESST-III [10], CRESST $\mathrm{LiAlO}_{2} \mathrm{G}$ [66], DarkSide-50 [67], PICASSO [68], CDMSlite [69], EDELWEISS surface [40], Collar [70], and PICO-60 [71] results. Here, our search extends to the low-mass WIMP of $200 \mathrm{MeV} / c^{2}$. Although our limits do not consider earth shielding effects [40,72,73], we found no significant impact on the upper limits using the VERNE code [74]. Because of the relatively higher threshold energy and background rates, our search cannot investigate the unexplored parameter space. This will be addressed in the future with improved detectors and analysis methods.

\section{SENSITIVITY FOR COSINE-200}

An effort to upgrade the ongoing COSINE-100 to the next-phase COSINE-200 has resulted in the production of $\mathrm{NaI}(\mathrm{Tl})$ crystals with reduced internal background from ${ }^{40} \mathrm{~K}$ and ${ }^{210} \mathrm{~Pb}[24,75,76]$ as well as an increased light yield of $22 \mathrm{NPE} / \mathrm{keV}$ [25]. The recrystallization method has achieved chemical purification of the raw NaI powder with sufficient reduction of $\mathrm{K}$ and $\mathrm{Pb}$ contamination [75,76]. A dedicated Kyropoulos grower for small test crystals has produced low-background $\mathrm{NaI}(\mathrm{Tl})$ crystals with reduced ${ }^{40} \mathrm{~K}$ and ${ }^{210} \mathrm{~Pb}$ of less than $20 \mathrm{ppb}$ and $0.5 \mathrm{mBq} / \mathrm{kg}$, respectively, corresponding to background rates of less than 1 counts/day/ $\mathrm{kg} / \mathrm{keVee}$ at the 1-6 keVee ROI [24]. A full-size Kyropoulos grower has been built for the $100 \mathrm{~kg}$-size crystal ingot and will provide approximately $200 \mathrm{~kg}$ of low-background $\mathrm{NaI}$ (Tl) detectors for the COSINE-200 experiment. The expected background level of those crystals is less than 0.5 counts/day/ $\mathrm{kg} / \mathrm{keVee}$ in the ROI as shown in Fig. 6. This estimate is based on the measured background levels for small test crystals as discussed in Ref. [24].

A high light yield of the $\mathrm{NaI}(\mathrm{Tl})$ crystal is crucial for enabling low-energy thresholds below 1 keVee. With an optimized concentration of thallium doping in the crystal, we achieved a high light yield of $17.1 \pm 0.5 \mathrm{NPE} / \mathrm{keVee}$, slightly larger than that of the COSINE-100 crystal of approximately $15 \mathrm{NPE} / \mathrm{keVee}$. Further increase in the light

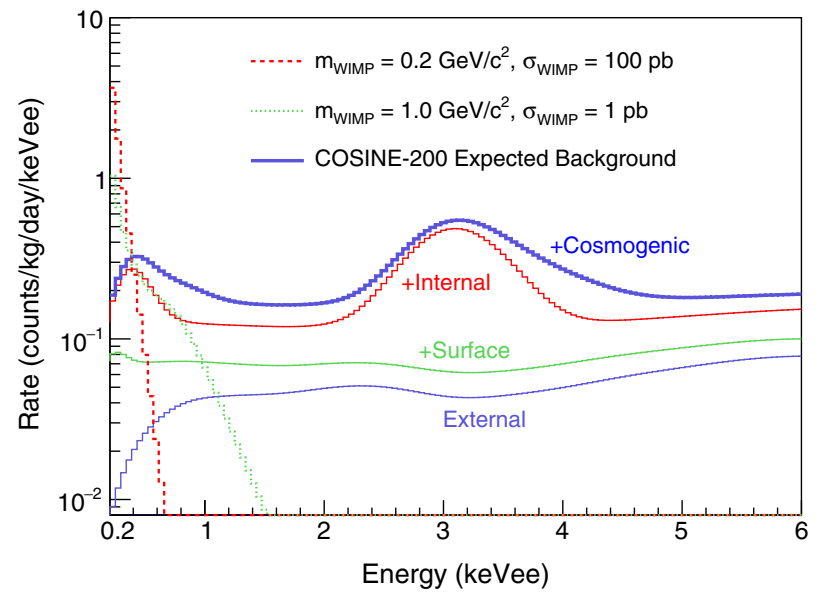

FIG. 6. Expected background spectrum (blue solid line) of the COSINE-200 crystal based on the developed low-background $\mathrm{NaI}(\mathrm{Tl})$ crystal [24] is compared with expected signals using the Migdal effect for WIMP-nucleon SI interactions of masses and cross sections of $0.2 \mathrm{GeV} / c^{2}, 100 \mathrm{pb}$ (red dashed line) and $1.0 \mathrm{GeV} / c^{2}, 1 \mathrm{pb}$ (green dotted line), respectively. A dominant background contribution is expected from the internal ${ }^{40} \mathrm{~K}$ and ${ }^{210} \mathrm{~Pb}$ remaining after purification. 
collection efficiency by $\sim 50 \%$ is possible with an improved encapsulation scheme as described in Ref. [25] that directly connects the crystal and PMTs without an intermediate quartz window for which a $22 \mathrm{NPE} / \mathrm{keVee}$ light yield is reported.

The typical trigger requirement of the COSINE-100 experiment is satisfied with coincident photoelectrons in two PMTs attached to each side of the crystal at approximately $0.13 \mathrm{keVee}$. However, the PMT-induced noise events are dominantly triggered below energies of a few keVee. The multivariable BDT provided a 1 keVee analysis threshold with less than $0.1 \%$ noise contamination and above $80 \%$ selection efficiency [22]. A key variable in the BDT is the likelihood parameter using the event shapes of the scintillation-like events and the PMT-induced noiselike events. A further improvement of the low-energy event selection is ongoing with the COSINE-100 data by developing new parameters for the BDT as well as employing a machine learning technique that uses raw waveforms directly. COSINE-200 targets an analysis threshold of 5 NPE (0.2 keVee), which is similar to the energy threshold that has already been achieved by the COHERENT experiment with $\mathrm{CsI}(\mathrm{Na})$ crystals [77].

The COSINE-200 experiment can be realized in a $4 \times 4$ array of $12.5 \mathrm{~kg} \mathrm{NaI}(\mathrm{Tl})$ modules by replacing crystals inside the COSINE-100 shield [26]. The COSINE-200 experiment will run at least three years for an unambiguous test of the DAMA/LIBRA annual modulation signals [78]. In addition, this detector can be used for general dark matter searches, especially for low-mass WIMPs via the Migdal process. Assuming the background reduction shown in Fig. 6, a high light yield of $22 \mathrm{NPE} / \mathrm{keVee}$, and an analysis threshold of $5 \mathrm{NPE}$ corresponding to $0.2 \mathrm{keVee}$, the sensitivities of the COSINE-200 experiment are evaluated below using the Migdal effect.

We generate the WIMP interaction signals including the Migdal effects as discussed above. Here we assume a one year data exposure with $200 \mathrm{~kg}$ crystals and the aforementioned detector performances. Poisson fluctuations of the measured NPE are considered for the detector resolution. Figure 7 presents the expected signal rate as a function of the WIMP mass for $1 \mathrm{~kg}$ of sodium and $1 \mathrm{~kg}$ of iodine for different interactions assuming $0.2 \mathrm{keVee}$ threshold. As one can see, sodium has an advantage for the lowmass WIMP searches with the Migdal process. In this scenario, the COSINE-200 experiment can probe the sub$\mathrm{GeV} / \mathrm{c}^{2}$ WIMP with mass down to $20 \mathrm{MeV} / \mathrm{c}^{2}$.

We use an ensemble of simulated experiments to estimate the sensitivity of the COSINE-200 experiment, expressed as the expected cross-section limits for the WIMP-nucleon SI and WIMP-proton SD interactions using the Migdal effect in the case of no signals. For each experiment, we determine a simulated spectrum for a background-only hypothesis with assumed background from Fig. 6. A Gaussian fluctuation of each background component and a poisson fluctuation of each energy bin are considered for each simulated experiment. We then fit the simulated data with a signal-plus-background hypothesis with flat priors for the signal and Gaussian constraints for the backgrounds based on understanding of the $\mathrm{NaI}(\mathrm{Tl})$ crystals [24,55]. Examples of signal spectra using the Migdal effect for SI interactions with WIMP masses (cross sections) of $0.2 \mathrm{GeV} / c^{2}(100 \mathrm{pb})$ and $1.0 \mathrm{GeV} / c^{2}(1 \mathrm{pb})$ are presented in Fig. 6. The same Bayesian approach is used for the single-hit energy spectra between 5 NPE (0.2 keVee) and 130 NPE (6 keVee) for each WIMP model for several masses. The $1 \sigma$ and $2 \sigma$ standard-deviation probability regions of the expected $90 \%$ C.L. limits are calculated from 2000 simulated experiments. Figure 8 shows those $1 \sigma$ and $2 \sigma$ regions, for the COSINE-200 experiment using the Migdal effect. The limits on the WIMP-nucleon SI interaction shown in Fig. 8(a) are compared with the current best limit on the low-mass WIMP searches of XENON1T with Migdal, DarkSide-50, and the expected sensitivities from future liquid Ar (TEALAB) [43], Xe [46], and Si [47] with Migdal. We also verify no significant impact on the upper limits using the VERNE code [74]. The COSINE-200 experiment has a potential to probe unexplored cross-section values for the WIMP mass below $200 \mathrm{MeV} / c^{2}$. In case of the WIMPproton SD interactions shown in Fig. 8(b), our projected sensitivities are compared with the current best limits from XENON1T with Migdal, PICO-60, CDMSlite [69], Collar [70], and PICASSO. Taking advantage of odd-proton numbers in both iodine and sodium, the projected sensitivities from the COSINE-200 experiments can probe

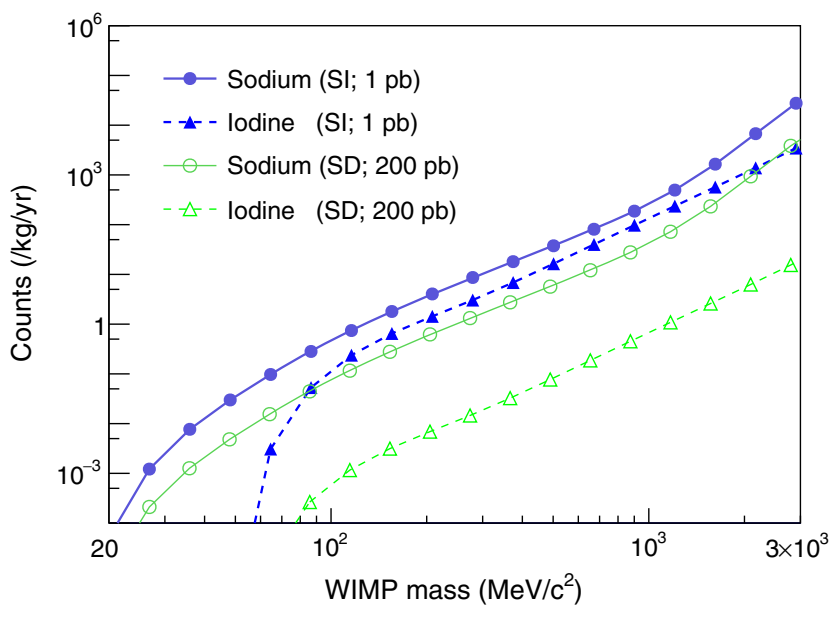

FIG. 7. The expected signal counts using the Migdal effect for a one year of data assuming the $0.2 \mathrm{keVee}$ analysis threshold are presented as a function of WIMP masses for SI (blue filled points) and SD interactions (green open points) for $1 \mathrm{~kg}$ of sodium (circle points) and $1 \mathrm{~kg}$ of iodine (triangle points). The cross sections for the SI and SD interactions are assumed to be 1 and $200 \mathrm{pb}$, respectively. 

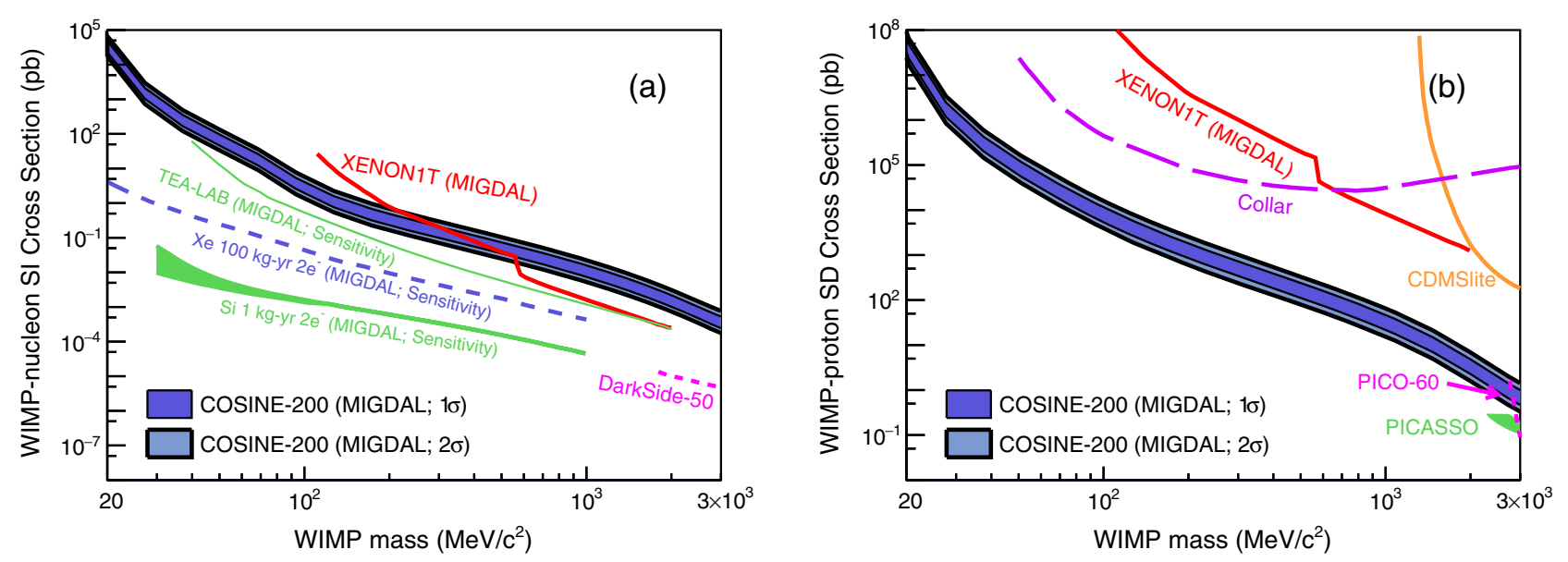

FIG. 8. COSINE-200 expected 90\% C.L. limits using the Migdal effect on the WIMP-nucleon SI cross section (a) and the WIMPproton SD cross section (b) are presented assuming the background-only hypothesis indicating the $1 \sigma$ and $2 \sigma$ standard deviation probability regions over which the limits have fluctuated. Those limits are compared with the current best limits from the XENON1T Migdal [42], DarkSide-50 [67], PICASSO [68], CDMSlite [69], Collar [70], and PICO-60 [71] together with the sensitivity limits from the future liquid Ar experiment (TEA-LAB) [43], Xe [46], and Si [47] with Migdal.

unexplored parameter spaces of WIMP masses below $2 \mathrm{GeV} / c^{2}$.

\section{CONCLUSION}

We consider the Migdal effect to search for the low-mass dark matter using the COSINE-100 data. With 1 keVee analysis threshold, our search extends down to $200 \mathrm{MeV} / c^{2}$ WIMP mass. We have investigated the expected sensitivities of the COSINE-200 experiment with a total mass of $200 \mathrm{~kg}$, a one-year period of stable operation, about 0.5 counts/day/keVee/kg background rate, and 0.2 keVee energy threshold. In this scenario, the COSINE-200 detector can explore low-mass dark matter down to $20 \mathrm{MeV} / c^{2}$, with a potential to probe currently unexplored parameter spaces for both SI and SD interactions.

\section{ACKNOWLEDGMENTS}

We thank the Korea Hydro and Nuclear Power (KHNP) Company for providing underground laboratory space at Yangyang. This work is supported by the Institute for Basic Science (IBS) under Project Codes No. IBS-R016-A1 and No. NRF-2021R1A2C3010989, Republic of Korea; NSF Grants No. PHY-1913742 and No. DGE1122492, WIPAC, the Wisconsin Alumni Research Foundation, United States; STFC Grants No. ST/N000277/1 and No. ST/K001337/1, United Kingdom; and Grant No. 2017/02952-0 FAPESP, CAPES Finance Code 001, CNPq 131152/2020-3, Brazil.
[1] D. Clowe, M. Bradač, A. H. Gonzalez, M. Markevitch, S. W. Randall, C. Jones, and D. Zaritsky, A direct empirical proof of the existence of dark matter, Astrophys. J. 648, L109 (2006).

[2] P. A. R. Ade et al. (Planck Collaboration), Planck 2015 results. XIII. Cosmological parameters, Astron. Astrophys. 594, A13 (2016).

[3] B. W. Lee and S. Weinberg, Cosmological Lower Bound on Heavy-Neutrino Masses, Phys. Rev. Lett. 39, 165 (1977).

[4] M. W. Goodman and E. Witten, Detectability of certain dark matter candidates, Phys. Rev. D 31, 3059 (1985).

[5] D. S. Akerib et al. (LUX Collaboration), Results from a Search for Dark Matter in the Complete LUX Exposure, Phys. Rev. Lett. 118, 021303 (2017).
[6] R. Agnese et al. (SuperCDMS Collaboration), Results from the Super Cryogenic Dark Matter Search Experiment at Soudan, Phys. Rev. Lett. 120, 061802 (2018).

[7] E. Aprile et al. (XENON Collaboration), First Dark Matter Search Results from the XENON1T Experiment, Phys. Rev. Lett. 119, 181301 (2017).

[8] P. Agnes et al. (DarkSide Collaboration), Low-Mass Dark Matter Search with the DarkSide-50 Experiment, Phys. Rev. Lett. 121, 081307 (2018).

[9] E. Aprile et al. (XENON Collaboration), Dark Matter Search Results from a One Ton-Year Exposure of XENON1T, Phys. Rev. Lett. 121, 111302 (2018).

[10] A. H. Abdelhameed et al. (CRESST Collaboration), First results from the CRESST-III low-mass dark matter program, Phys. Rev. D 100, 102002 (2019). 
[11] P. Zyla et al. (Particle Data Group Collaboration), Review of particle physics, Prog. Theor. Exp. Phys. 2020, 083C01 (2020).

[12] R. Essig et al., Working group report: New light weakly coupled particles, in Proceedings of the 2013 Community Summer Study on the Future of U.S. Particle Physics: Snowmass on the Mississippi (CSS2013): Minneapolis, MN, 2013, arXiv:1311.0029.

[13] E. Aprile et al. (XENON Collaboration), Low-mass dark matter search using ionization signals in XENON100, Phys. Rev. D 94, 092001 (2016); Phys. Rev. D 95, 059901(E) (2017).

[14] E. Aprile et al. (XENON Collaboration), Light Dark Matter Search with Ionization Signals in XENON1T, Phys. Rev. Lett. 123, 251801 (2019).

[15] A. Aguilar-Arevalo et al. (DAMIC Collaboration), Constraints on Light Dark Matter Particles Interacting with Electrons from DAMIC at SNOLAB, Phys. Rev. Lett. 123, 181802 (2019).

[16] Q. Arnaud et al. (EDELWEISS Collaboration), First Germanium-Based Constraints on sub-MeV Dark Matter with the EDELWEISS Experiment, Phys. Rev. Lett. 125, 141301 (2020).

[17] L. Barak et al. (SENSEI Collaboration), SENSEI: DirectDetection Results on sub-GeV Dark Matter from a New Skipper-CCD, Phys. Rev. Lett. 125, 171802 (2020).

[18] I. Alkhatib et al. (SuperCDMS Collaboration), Light Dark Matter Search with a High-Resolution Athermal Phonon Detector Operated Above Ground, Phys. Rev. Lett. 127, 061801 (2021).

[19] C. Cheng et al. (PandaX-II Collaboration), Search for Light Dark Matter-Electron Scatterings in the PandaX-II Experiment, Phys. Rev. Lett. 126, 211803 (2021).

[20] A. Migdal, Ionization of atoms accompanying $\alpha$ - and $\beta$ decay, J. Phys. USSR 4, 449 (1941).

[21] M. Ibe, W. Nakano, Y. Shoji, and K. Suzuki, Migdal effect in dark matter direct detection experiments, J. High Energy Phys. 03 (2018) 194.

[22] G. Adhikari et al. (COSINE-100 Collaboration), Lowering the energy threshold in COSINE-100 dark matter searches, Astropart. Phys. 130, 102581 (2021).

[23] G. Adhikari et al. (COSINE-100 Collaboration), Strong constraints from COSINE-100 on the DAMA dark matter results using the same sodium iodide target, Sci. Adv. 7, eabk2699 (2021).

[24] B. J. Park et al. (COSINE Collaboration), Development of ultrapure $\mathrm{NaI}(\mathrm{Tl})$ detectors for the COSINE-200 experiment, Eur. Phys. J. C 80, 814 (2020).

[25] J. J. Choi, B. J. Park, C. Ha, K. W. Kim, S. K. Kim, Y. D. Kim, Y. J. Ko, H. S. Lee, S. H. Lee, and S. L. Olsen, Improving the light collection using a new $\mathrm{NaI}(\mathrm{Tl})$ crystal encapsulation, Nucl. Instrum. Methods Phys. Res., Sect. A 981, 164556 (2020).

[26] G. Adhikari et al. (COSINE-100 Collaboration), Initial performance of the COSINE-100 experiment, Eur. Phys. J. C 78, 107 (2018).

[27] H. S. Lee et al. (Kims Collaboration), First limit on WIMP cross section with low background $\mathrm{CsI}(\mathrm{Tl})$ crystal detector, Phys. Lett. B 633, 201 (2006).
[28] S. C. Kim et al., New Limits on Interactions between Weakly Interacting Massive Particles and Nucleons Obtained with CsI(Tl) Crystal Detectors, Phys. Rev. Lett. 108, 181301 (2012).

[29] H. Prihtiadi et al. (COSINE-100 Collaboration), Muon detector for the COSINE-100 experiment, J. Instrum. 13, T02007 (2018).

[30] H. Kim et al., The environmental monitoring system at the COSINE-100 experiment, J. Instrum. 17, T01001 (2021).

[31] J. S. Park, P. Adhikari, G. Adhikari, S. Y. Oh, N. Y. Kim, Y. D. Kim, C. Ha, K. S. Park, H. S. Lee, and E. J. Jeon (KIMS Collaboration), Performance of a prototype active veto system using liquid scintillator for a dark matter search experiment, Nucl. Instrum. Methods Phys. Res., Sect. A 851, 103 (2017).

[32] G. Adhikari et al., The COSINE-100 liquid scintillator veto system, Nucl. Instrum. Methods Phys. Res., Sect. A 1006, 165431 (2021).

[33] H. Prihtiadi et al. (COSINE-100 Collaboration), Measurement of the cosmic muon annual and diurnal flux variation with the COSINE-100 detector, J. Cosmol. Astropart. Phys. 02 (2021) 013.

[34] G. Adhikari et al. (COSINE-100 Collaboration), The COSINE-100 data acquisition system, J. Instrum. 13, P09006 (2018).

[35] A. Manzur, A. Curioni, L. Kastens, D. N. McKinsey, K. Ni, and T. Wongjirad, Scintillation efficiency and ionization yield of liquid xenon for mono-energetic nuclear recoils down to $4 \mathrm{keV}$, Phys. Rev. C 81, 025808 (2010).

[36] J.H. Lee et al., Measurement of the quenching and channeling effects in a CsI crystal used for a WIMP search, Nucl. Instrum. Methods Phys. Res., Sect. A 782, 133 (2015).

[37] H. W. Joo, H. S. Park, J. H. Kim, S. K. Kim, Y. D. Kim, H. S. Lee, and S. H. Kim, Quenching factor measurement for $\mathrm{NaI}(\mathrm{Tl})$ scintillation crystal, Astropart. Phys. 108, 50 (2019).

[38] M. Kimura, M. Tanaka, T. Washimi, and K. Yorita, Measurement of the scintillation efficiency for nuclear recoils in liquid argon under electric fields up to 3 kV/cm, Phys. Rev. D 100, 032002 (2019).

[39] D. S. Akerib et al. (LUX Collaboration), Results of a Search for Sub-GeV Dark Matter Using 2013 LUX Data, Phys. Rev. Lett. 122, 131301 (2019).

[40] E. Armengaud et al. (EDELWEISS Collaboration), Searching for low-mass dark matter particles with a massive Ge bolometer operated above-ground, Phys. Rev. D 99, 082003 (2019).

[41] Z. Z. Liu et al. (CDEX Collaboration), Constraints on SpinIndependent Nucleus Scattering with sub-GeV Weakly Interacting Massive Particle Dark Matter from the CDEX-1B Experiment at the China Jinping Underground Laboratory, Phys. Rev. Lett. 123, 161301 (2019).

[42] E. Aprile et al. (XENON Collaboration), Search for Light Dark Matter Interactions Enhanced by the Migdal Effect or Bremsstrahlung in XENON1T, Phys. Rev. Lett. 123, 241803 (2019).

[43] G. Grilli di Cortona, A. Messina, and S. Piacentini, Migdal effect and photon bremsstrahlung: Improving the sensitivity 
to light dark matter of liquid argon experiments, J. High Energy Phys. 11 (2020) 034.

[44] C. Savage, G. Gelmini, P. Gondolo, and K. Freese, Compatibility of DAMA/LIBRA dark matter detection with other searches, J. Cosmol. Astropart. Phys. 04 (2009) 010.

[45] C. Kouvaris and J. Pradler, Probing sub-GeV Dark Matter with Conventional Detectors, Phys. Rev. Lett. 118, 031803 (2017).

[46] R. Essig, J. Pradler, M. Sholapurkar, and T.-T. Yu, Relation between the Migdal Effect and Dark Matter-Electron Scattering in Isolated Atoms and Semiconductors, Phys. Rev. Lett. 124, 021801 (2020).

[47] S. Knapen, J. Kozaczuk, and T. Lin, Migdal Effect in Semiconductors, Phys. Rev. Lett. 127, 081805 (2021).

[48] G. Adhikari et al. (COSINE-100 Collaboration), Search for a Dark Matter-Induced Annual Modulation Signal in NaI (Tl) with the COSINE-100 Experiment, Phys. Rev. Lett. 123, 031302 (2019).

[49] G. Adhikari et al. (COSINE-100 Collaboration), An experiment to search for dark-matter interactions using sodium iodide detectors, Nature (London) 564, 83 (2018).

[50] G. Adhikari et al. (COSINE-100 and Sogang Phenomenology Group Collaboration), COSINE-100 and DAMA/LIBRA-phase2 in WIMP effective models, J. Cosmol. Astropart. Phys. 06 (2019) 048.

[51] J. H. Friedman, Greedy function approximation: A gradient boosting machine, Ann. Stat. 29, 1189 (2001).

[52] S. Agostinelli et al. (GEANT4 Collaboration), GEANT4: A simulation toolkit, Nucl. Instrum. Methods Phys. Res., Sect. A 506, 250 (2003).

[53] G. Adhikari et al. (KIMS Collaboration), Understanding $\mathrm{NaI}(\mathrm{Tl})$ crystal background for dark matter searches, Eur. Phys. J. C 77, 437 (2017).

[54] P. Adhikari et al. (COSINE-100 Collaboration), Background model for the $\mathrm{NaI}(\mathrm{Tl})$ crystals in COSINE-100, Eur. Phys. J. C 78, 490 (2018).

[55] G. Adhikari et al. (COSINE-100 Collaboration), Background modeling for dark matter search with 1.7 years of COSINE-100 data, Eur. Phys. J. C 81, 837 (2021).

[56] J. Lewin and P. Smith, Review of mathematics, numerical factors, and corrections for dark matter experiments based on elastic nuclear recoil, Astropart. Phys. 6, 87 (1996).

[57] K. Freese, M. Lisanti, and C. Savage, Colloquium: Annual modulation of dark matter, Rev. Mod. Phys. 85, 1561 (2013).

[58] V. A. Bednyakov and F. Simkovic, Nuclear spin structure in dark matter search: The finite momentum transfer limit, Phys. Part. Nucl. 37, S106 (2006).

[59] M. I. Gresham and K. M. Zurek, Effect of nuclear response functions in dark matter direct detection, Phys. Rev. D 89, 123521 (2014).

[60] V. Gluscevic and S. D. McDermott, dmdd: Dark matter direct detection, Astrophysics Source Code Library [ascl:1506.002], 2015.

[61] V. Gluscevic, M. I. Gresham, S. D. McDermott, A. H. G. Peter, and K. M. Zurek, Identifying the Theory of Dark
Matter with Direct Detection, J. Cosmol. Astropart. Phys. 12 (2015) 057.

[62] N. Anand, A. L. Fitzpatrick, and W. C. Haxton, Weakly interacting massive particle-nucleus elastic scattering response, Phys. Rev. C 89, 065501 (2014).

[63] A. L. Fitzpatrick, W. Haxton, E. Katz, N. Lubbers, and Y. $\mathrm{Xu}$, The effective field theory of dark matter direct detection, J. Cosmol. Astropart. Phys. 02 (2013) 004.

[64] J. Lindhard, V. Nielsen, M. Scharff, and P. Thomsen, Integral equations governing radiation effects. (Notes on Atomic collisions, III), Mat. Fys. Medd. K. Dan. Vidensk. Selsk 33, 1 (1963).

[65] Y. J. Ko et al. (COSINE-100 Collaboration), Comparison between DAMA/LIBRA and COSINE-100 in the light of Quenching Factors, J. Cosmol. Astropart. Phys. 11 (2019) 008.

[66] A. H. Abdelhameed et al. (CRESST Collaboration), Cryogenic characterization of a $\mathrm{LiAlO}_{2}$ crystal and new results on spin-dependent dark matter interactions with ordinary matter, Eur. Phys. J. C 80, 834 (2020).

[67] P. Agnes et al. (DarkSide Collaboration), Low-Mass Dark Matter Search with the DarkSide-50 Experiment, Phys. Rev. Lett. 121, 081307 (2018).

[68] E. Behnke et al., Final results of the PICASSO dark matter search experiment, Astropart. Phys. 90, 85 (2017).

[69] R. Agnese et al. (SuperCDMS Collaboration), Low-mass dark matter search with CDMSlite, Phys. Rev. D 97, 022002 (2018).

[70] J. I. Collar, Search for a nonrelativistic component in the spectrum of cosmic rays at Earth, Phys. Rev. D 98, 023005 (2018).

[71] C. Amole et al. (PICO Collaboration), Dark matter search results from the complete exposure of the PICO-60 $\mathrm{C}_{3} \mathrm{~F}_{8}$ bubble chamber, Phys. Rev. D 100, 022001 (2019).

[72] C. Kouvaris, Earth's stopping effect in directional dark matter detectors, Phys. Rev. D 93, 035023 (2016).

[73] B. J. Kavanagh, Earth scattering of superheavy dark matter: Updated constraints from detectors old and new, Phys. Rev. D 97, 123013 (2018).

[74] B. Kavanagh, bradkav/verne:v1.2, https://github.com/ bradkav/verne/tree/v1.2, 2015.

[75] K. Shin, O. Gileva, Y. Kim, H. S. Lee, and H. Park, Reduction of the radioactivity in sodium iodide (NaI) powder by recrystallization method, J. Radioanal. Nucl. Chem. 317, 1329 (2018).

[76] K. Shin, J. Choe, O. Gileva, A. Iltis, Y. Kim, C. Lee, H. S. Lee, M.H. Lee, and H. K. Park, A facility for mass production of ultrapure NaI powder for the COSINE-200 experiment, J. Instrum. 15, C07031 (2020).

[77] D. Akimov et al. (COHERENT Collaboration), Observation of coherent elastic neutrino-nucleus scattering, Science 357, 1123 (2017).

[78] P. Adhikari et al., Understanding internal backgrounds in $\mathrm{NaI}(\mathrm{Tl})$ crystals toward a $200 \mathrm{~kg}$ array for the KIMS-NaI experiment, Eur. Phys. J. C 76, 185 (2016). 